\title{
C66 ameliorates diabetic nephropathy in mice by both upregulating NRF2 function via increase in miR-200a and inhibiting miR-21
}

\author{
Hao Wu ${ }^{1,3} \cdot$ Lili Kong ${ }^{2,3} \cdot$ Yi Tan ${ }^{3,4} \cdot$ Paul N. Epstein ${ }^{3} \cdot$ Jun Zeng ${ }^{3} \cdot J_{u n l i a n}$ Gu$^{3}$ •

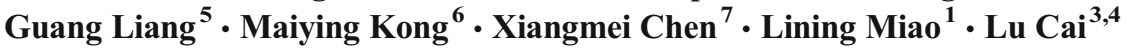

Received: 5 January 2016/Accepted: 23 March 2016 / Published online: 26 April 2016

(C) Springer-Verlag Berlin Heidelberg 2016

\begin{abstract}
Aims/hypothesis Diabetic nephropathy is the leading cause of end-stage renal disease. Previously we reported that C66, a novel analogue of curcumin with a very high bioavailability, ameliorated diabetic nephropathy in mice, with little known about the mechanism. The present study aimed to define the mechanism by which C66 ameliorates diabetic nephropathy. Methods Our aim was to discover whether C66 acts through the activation of nuclear factor (erythroid-derived 2)-like 2 (NFE2L2 or NRF2), which governs the antioxidant response.
\end{abstract}

Lining Miao

miaolining55@163.com

Lu Cai

10cai001@louisville.edu

1 Department of Nephrology, the Second Hospital of Jilin University, 218 Ziqiang St, Changchun, Jilin 130041, People's Republic of China

2 Department of Nephrology, the First Hospital of Jilin University, Changchun, Jilin, People's Republic of China

3 Kosair Children's Hospital Research Institute at the Department of Pediatrics, Wendy L. Novak Diabetes Care Center, University of Louisville, 570 S Preston St, Baxter I., Louisville, KY 40202, USA

4 Chinese-American Research Institute for Diabetic Complications, School of Pharmaceutical Science, Wenzhou Medical University, Wenzhou, Zhejiang, People's Republic of China

5 Chemical Biology Research Center, School of Pharmaceutical Science, Wenzhou Medical University, Wenzhou, Zhejiang, People's Republic of China

6 Department of Bioinformatics and Biostatistics, SPHIS, University of Louisville, Louisville, KY, USA

7 Department of Nephrology, Chinese PLA General Hospital, Beijing, People's Republic of China
Streptozotocin-induced $\mathrm{Nrf2}$ (also known as $\mathrm{Nfe} 2 \mathrm{l2}$ )-knockout and wild-type (WT) diabetic mice were treated with C66. To determine whether the actions of $\mathrm{C} 66$ on NRF2 are mediated by microRNA (miR)-200a, WT diabetic mice were treated with C66 in the presence or absence of an in vivo miR-200a inhibitor (locked nucleic acid-modified anti-miR-200a [LNA200a]) for 6 months. To determine whether miR-21 downregulation provided an NRF2-independent basis for C66 protection, Nrf2-knockout diabetic mice were treated with either C66 or an inhibitor of miR-21 (locked nucleic acid-modified anti-miR-21 [LNA-21]).

Results Deletion of Nrf2 partially abolished diabetic nephropathy protection by C66, confirming the requirement of NRF2 for this protection. Diabetic mice, but not C66-treated diabetic mice, developed significant albuminuria, renal oxidative damage and fibrosis. C66 upregulated renal miR-200a, inhibited kelch-like ECH-associated protein 1 and induced NRF2 function, effects that were prevented by LNA-200a. However, LNA-200a only partially reduced the protection afforded by C66, suggesting the existence of miR-200a/NRF2-independent mechanisms for $\mathrm{C} 66$ protection. C66 was also found to inhibit diabetes induction of miR-21. Both C66 and LNA-21 produced similar reductions in miR-21, albuminuria and renal fibrosis. Conclusions/interpretation The present study indicates that in addition to upregulating NRF2 by increasing miR-200a, C66 also protects against diabetic nephropathy by inhibiting miR-21.

Keywords Albuminuria - Curcumin - Diabetic nephropathy · Fibrosis $\cdot$ KEAP1 $\cdot$ miR-200 $\cdot$ miR-21 $\cdot$ NRF2

$\begin{array}{ll}\text { Abbreviations } & \\ \text { 3-NT } & \text { 3-Nitrotyrosine } \\ \text { 4-HNE } & \text { 4-Hydroxynonenal } \\ \text { CA } & \text { Cinnamic aldehyde }\end{array}$




$\begin{array}{ll}\text { COL4 } & \text { Collagen 4 } \\ \text { DTT } & \text { Dithiothreitol } \\ \text { FN } & \text { Fibronectin } \\ \text { JNK } & \text { c-Jun N-terminal kinase } \\ \text { KEAP1 } & \text { Kelch-like ECH-associated protein 1 } \\ \text { LNA-200a } & \text { Locked nucleic acid-modified anti-miR- } \\ & \text { 200a } \\ \text { LNA-21 } & \text { Locked nucleic acid-modified anti-miR-21 } \\ \text { miR } & \text { microRNA } \\ \text { NFE2L2, NRF2 } & \text { Nuclear factor (erythroid-derived 2)-like 2 } \\ \text { PAS } & \text { Periodic acid-Schiff } \\ \text { PDCD4 } & \text { Programmed cell death protein 4 } \\ \text { pri-miR-21 } & \text { Primary-miR-21 } \\ \text { SFN } & \text { Sulforaphane } \\ \text { STZ } & \text { Streptozotocin } \\ \text { UACR } & \text { Urinary albumin-to-creatinine ratio } \\ \text { WT } & \text { Wild-type }\end{array}$

\section{Introduction}

Diabetic nephropathy is the leading cause of end-stage renal disease [1], resulting in high mortality. As a long-term complication of diabetes, diabetic nephropathy still cannot be effectively treated and so there is an urgent need to develop more effective medicines to prevent or slow down its progression [2].

Oxidative stress contributes to the pathogenesis of diabetic nephropathy [3]. Nuclear factor (erythroid-derived 2)-like 2 (NFE2L2 or NRF2) controls cellular defence mechanisms against oxidative stress [4] by turning on transcription of antioxidant genes, such as $\mathrm{Hol}$ and $\mathrm{NqO}$ [5]. NRF2 has been demonstrated to play a key role in protection from diabetic nephropathy $[2,3]$. Curcumin is a natural compound that induces NRF2 [6] and prevents diabetes-induced renal injury in animal models [7, 8]. Although curcumin has beneficial effects in animal models of many diseases, poor bioavailability limits its clinical application. The doses of curcumin used in animal models of diabetic nephropathy range from $50 \mathrm{mg} / \mathrm{kg}$ to $200 \mathrm{mg} / \mathrm{kg}$ daily [7, 9-11]. C66 is a novel curcumin analogue with a much lower effective dose of $5 \mathrm{mg} / \mathrm{kg}$ administered every other day, and has been shown to establish protection from diabetic nephropathy and diabetic cardiomyopathy $[12,13]$. We have also demonstrated that $\mathrm{C} 66$ activated NRF2 in diabetic aorta [14]. However, it is not certain that activation of NRF2 is necessary for C66 to provide protection against diabetic nephropathy. Therefore our first question to address is whether renal protection by C66 in diabetes is mediated by NRF2.

Kelch-like ECH-associated protein 1 (KEAP1) sequesters NRF2 in the cytoplasm, promoting its proteasomal degradation [3]. Thus, one strategy for increasing the activity of NRF2 is to reduce cytoplasmic KEAP1. MicroRNAs (miRs) are short noncoding RNAs that modulate gene expressions [15]. miR-200a ameliorates diabetic nephropathy and promotes degradation of
Keap1 mRNA [16]. In diabetic mice, suppression of renal miR200a increases KEAP1, decreases NRF2 and worsens renal fibrosis and albuminuria [17]. Interestingly, a difluorinated analogue of curcumin upregulates expression of the miR-200 family $[18,19]$. Curcumin analogue C66 may also upregulate miR-200a which could mediate the ability of C66 to upregulate NRF2 and prevent diabetic nephropathy. miR-21 also has a key role in the pathogenesis of diabetic nephropathy [20-22] but unlike miR-200a, it exacerbates diabetic nephropathy. Curcumin downregulates miR-21 [18, 23] and this likely contributes to the beneficial effect of curcumin against diabetic nephropathy. C66 has not been tested for its ability to modulate renal levels of miR-200a or miR-21; this could be an essential step in the pathway by which $\mathrm{C} 66$ provides protection from diabetic nephropathy.

The present study was performed to determine the mechanism(s) by which C66 provides protection against diabetic nephropathy by answering the following questions: (1) does prevention of diabetic nephropathy by C66 require NRF2?; (2) does C66 upregulate miR-200a or downregulate miR-21?; (3) is C66 upregulation of NRF2 mediated by miR-200a? and (4) is C66 modulation of either miR-21 or miR-200a sufficient to protect against diabetic nephropathy? To these ends, $N r f 2$ (also known as Nfe2l2)-knockout ( $N r f 2$-null) mice and their wild-type (WT) counterparts were used with and without C66 treatment, and in combination with specific in vivo inhibitors of miR-200a or miR-21.

\section{Methods}

Animal treatment $\mathrm{Nrf2}$-null $\left(N r f 2^{-/}\right)$male mice with C57BL/6J background (WT) were obtained through breeding of homozygote $\left(\mathrm{Nrf2}^{-/}\right)$with heterozygote $\left(\mathrm{Nrf2}^{+/}\right)$following the mating system suggested by Jackson Laboratory (Bar Harbor, ME, USA). C57BL/6J male mice $\left(\mathrm{Nrf}^{+/+}\right)$were also purchased from the Jackson Laboratory. All mice were housed in University of Louisville Research Resources Center at $22^{\circ} \mathrm{C}$, on a $12 \mathrm{~h}$ light-dark cycle, with free access to standard rodent feed and tap water. The Institutional Animal Care and Use Committee at University of Louisville approved all experimental procedures for these animals, and all procedures complied with the Guide for the Care and Use of Laboratory Animals by the US National Institutes of Health (2011, eighth edition). To test the role of NRF2 in C66 protection against diabetic nephropathy, 8-week-old male WT mice were randomised into the following four groups $(n=7)$ : control (Ctrl), control treated with $\mathrm{C} 66$ (Ctrl/C66), diabetes (DM) and diabetes treated with $\mathrm{C} 66$ (DM/C66). To further explore whether miR-200a mediates NRF2 activation by C66, 8-week-old male WT mice were randomised into six groups $(n=7)$ : $\mathrm{Ctrl}, \mathrm{Ctrl} / \mathrm{C} 66, \mathrm{DM}$, DM/C66, C66/locked nucleic acid-modified anti-miR-200a (LNA-200a) negative control co-treated DM (DM/C66/LNA- 
200a-NC) and C66/LNA-200a co-treated DM (DM/C66/LNA200a). To explore whether the inhibition of miR-21 by C66 provides additional protection against diabetic nephropathy in the absence of NRF2, 8-week-old male Nrf2-null mice were randomised into six groups $(n=7): \mathrm{Ctrl}, \mathrm{Ctrl} / \mathrm{C} 66, \mathrm{DM}, \mathrm{DM} /$ C66, locked nucleic acid-modified anti-miR-21 (LNA-21) negative control treated DM (DM/LNA-21-NC) and LNA-21 treated DM (DM/LNA-21). Mice received an intraperitoneal injection of either sodium citrate or streptozotocin (STZ) (SigmaAldrich, St Louis, MO, USA; $50 \mathrm{mg} / \mathrm{kg}$, dissolved in $0.1 \mathrm{~mol} / \mathrm{l}$ sodium citrate, $\mathrm{pH} 4.5$ ) on five consecutive days. One week after the last injection of STZ, fasting glucose levels ( $4 \mathrm{~h}$ fast) were measured and mice with a fasting glucose level above $13.89 \mathrm{mmol} / \mathrm{l}$ were considered diabetic. Diabetic mice and age-matched controls were then treated by gavage with C66 (5 mg/kg, dissolved in 1\% carboxymethyl cellulose-Na solution) or vehicle every other day for 24 weeks. LNA-200a, LNA-21 and their respective mismatched controls (Exiqon, Woburn, MA, USA; $2 \mathrm{mg} / \mathrm{kg}$, dissolved in normal saline [154 mmol/1 NaCl]) were delivered subcutaneously at the same time as C66, twice weekly for 24 weeks. Blood glucose levels were recorded on days 28, 56, 84, 112, 140 and 168 after diabetes onset. Mice were then killed and their kidneys taken

Fig. 1 Deletion of the $N r f 2$ gene partially abolished the renal protection afforded by C66 against diabetes-induced albuminuria. (a, b) Blood glucose levels in C57 WT mice (a) and Nrf2-null mice (b) were monitored at $0,4,8,12,16,20$ and 24 weeks after diabetes onset. (c) Systolic BP was recorded at 24 weeks. (d) Body weight was measured at 0 and 24 weeks (W). (e) UACR in C57 WT and Nrf2null mice at 24 weeks. (f, g) Protein levels of TGF- $\beta 1$ (f) and $\mathrm{FN}$ (g) were evaluated by western blot. Ctrl, control. Black squares and white bars, Ctrl; red circles and light grey bars, Ctrl treated with C66; blue triangles and dark grey bars, diabetes; pink inverted triangles and black bars, diabetes treated with C66. Data were normalised by $\mathrm{Ctrl}$ and presented as means $\pm \mathrm{SD}(n=7) .{ }^{*} p<0.05$ vs control; ${ }^{\dagger} p<0.05$ vs diabetes; ${ }^{\ddagger} p<0.05$ vs WT diabetes; ${ }^{\S} p<0.05$ vs WT control for analysis. Experimenters of this study were blind to group assignment and outcome assessment.

Analysis of kidney function Urine albumin and creatinine were measured on a spot urine sample by a mouse albumin ELISA kit (Bethyl Laboratories, Montgomery, TX, USA) and a QuantiChrom Creatinine Assay Kit (BioAssay Systems, Hayward, CA, USA). The urinary albumin-to-creatinine ratio (UACR) was calculated.

Measurement of blood pressure Systolic BP was recorded on day 168 after the onset of diabetes with a non-invasive tail cuff Plethysmograph system (CODA6TM; Kent Scientific, Torrington, CT, USA) as previously described [24].

Western blot analysis Western blot was performed using kidney cortex as described in our previous study [25]. The primary antibodies were anti-KEAP1 (Santa Cruz Biotechnology, Dallas, TX, USA; 1:1,000), anti-NRF2 (Santa Cruz Biotechnology; 1:1,000), anti-Histone H3 (Santa Cruz Biotechnology; 1:500), anti-4-HNE (Alpha Diagnostic, San Antonio, TX, USA; 1:3, 000), anti-3-NT (Millipore, Temecula, CA, USA; 1:1,000), anti-TGF- $\beta 1$ (Cell Signaling, Beverly, MA, USA; 1:500), b
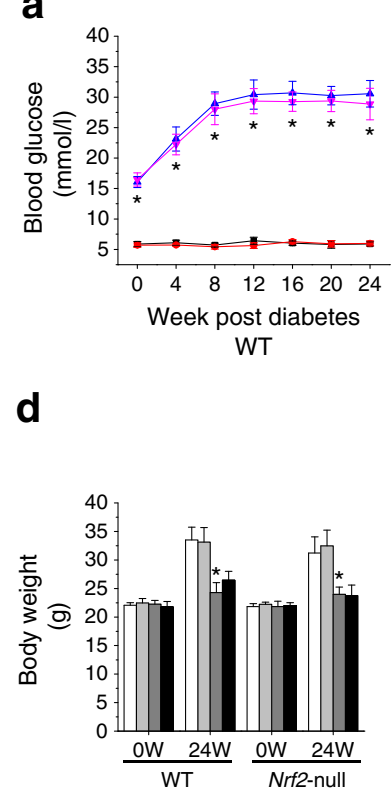

e

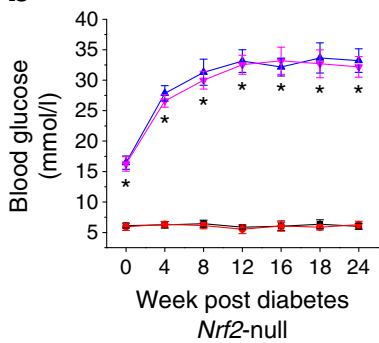

C

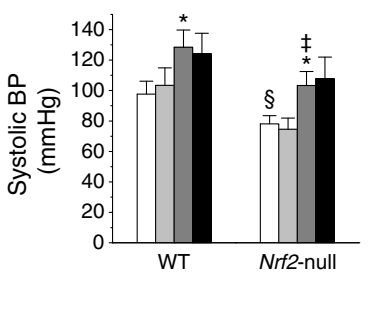

f

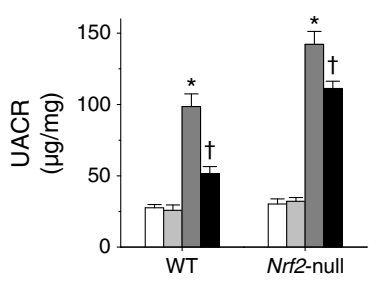

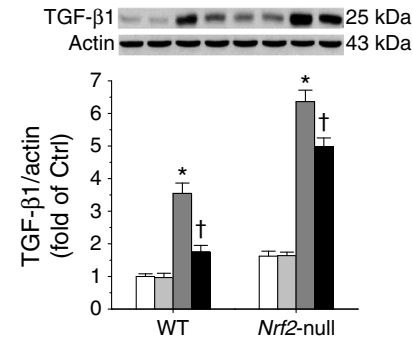

g

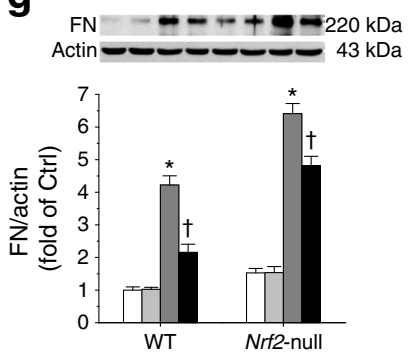


anti-COL4 (Abcam, Cambridge, MA, USA, 1:500), anti-FN (Santa Cruz Biotechnology; 1:500), anti-Smad7 (Santa Cruz Biotechnology; 1:1,000), anti-Smad3 (Santa Cruz Biotechnology; 1:1,000), anti-p-Smad3 (Cell Signaling; 1:500), anti-p-JNK (Cell Signaling; 1:500), anti-PDCD4 (Santa Cruz Biotechnology; 1:1,000), anti-t-JNK (Cell Signaling; 1:1,000), anti-Actin (Santa Cruz Biotechnology; 1:2,000) and antiGAPDH (Santa Cruz Biotechnology; 1:3,000). These antibodies were routinely validated when they arrived from suppliers with previous positive tissues that had been defined either based on the knockout or overexpression of the target protein.

\section{Quantitative reverse transcription PCR}

Quantitative RT-PCR (qRT-PCR) was performed as described in our previous studies $[26,27]$. qRT-PCR for miRNAs was performed with the TaqMan MicroRNA Reverse Transcription Kit (Life Technologies, Grand Island, NY, USA). Primers for Keap 1, NAD(P)H quinone oxidoreductase (Nqol), haem oxygenase-1 (Hol), primary-miR-21 (pri-miR-21), miR-21, Smad7, programmed cell death protein 4 (Pdcd4), actin and U6 were all purchased from Life Technologies.

Histological and immunohistochemical staining Kidney tissues were fixed immediately in $10 \%$ buffered formalin solution after harvesting and were embedded in paraffin and sectioned into $5 \mu \mathrm{m}$ thick sections onto glass slides. The sections were processed for periodic acid-Schiff (PAS), Masson's trichrome and immunohistochemical staining using antibody against NRF2 (Santa Cruz Biotechnology; 1:100 dilution).

Isolation of nuclei The renal nuclei were isolated using a nuclei isolation kit (Sigma-Aldrich). Kidney tissue $(30 \mathrm{mg}$ )
Fig. 2 C66 upregulation of renal NRF2 requires an increase in miR-200a. (a-e) The effects of C66, diabetes, LNA-200a and their combinations were compared on RNA levels of miR200a (a) and Keapl (b) and on protein levels of KEAP1 (c), total NRF2 (d) and nuclear NRF2 (e). (f) Immunohistochemical staining for NRF2 (Ctrl, control; DM, diabetes). Scale bar, $50 \mu \mathrm{m}$. (g, h) RNA levels of $\mathrm{Nqol}$ (g) and $\mathrm{Hol}$ (h). Data were normalised by Ctrl and presented as means $\pm \mathrm{S} \mathrm{D}$ $(n=7) .{ }^{*} p<0.05$ vs control; ${ }^{\dagger} p<0.05$ vs diabetes; ${ }^{\star} p<0.05$ vs DM/C66/LNA-200a-NC a

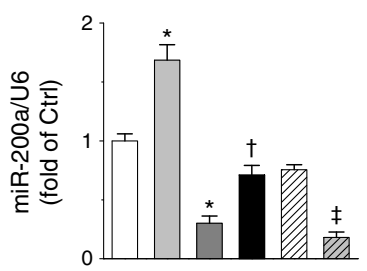

b
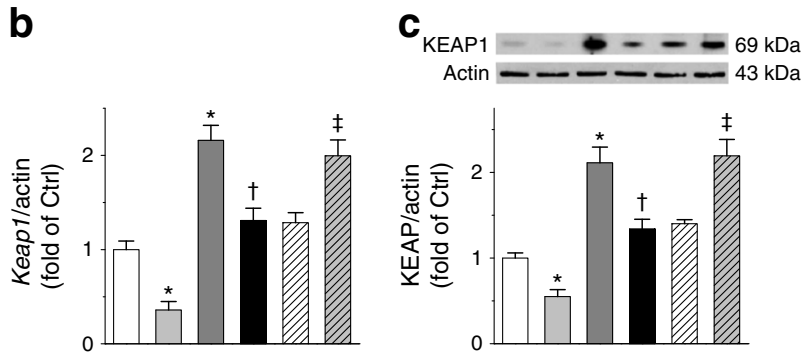

d

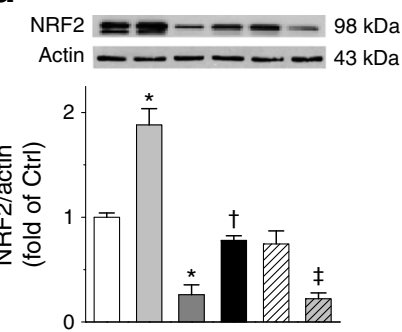

e
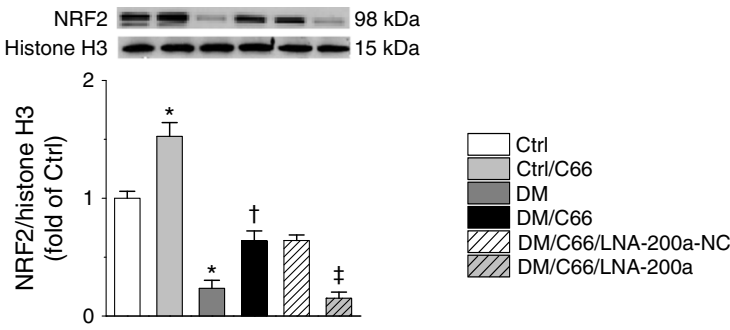

f $\mathrm{Ctrl}$ Ctrl/C66 DM

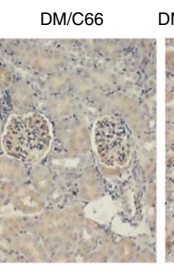

DM/C66/LNA-200a-NC DM/C66/LNA-200a
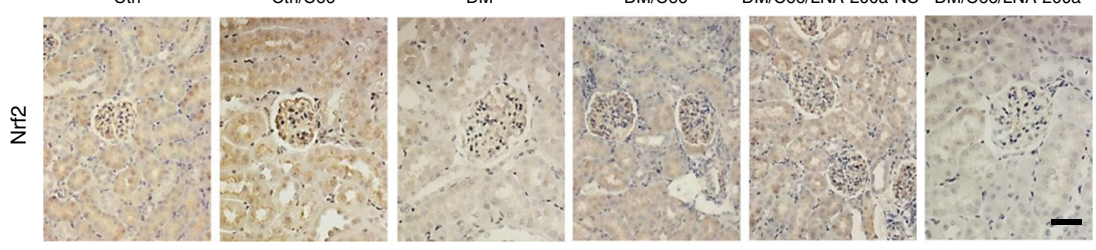

g

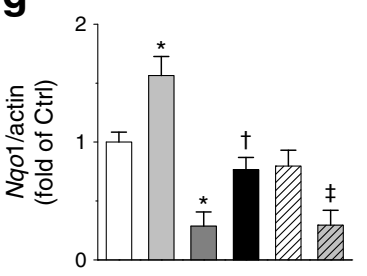

h

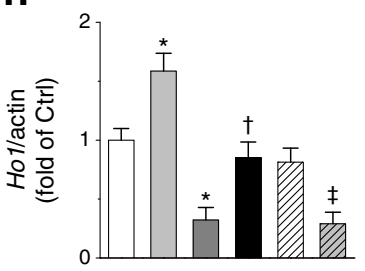


from each mouse was homogenised for $45 \mathrm{~s}$ in $150 \mu$ l of cold lysis buffer containing $0.5 \mu \mathrm{l}$ of dithiothreitol (DTT) and $0.1 \%$ Triton X-100. After that, $300 \mu \mathrm{l}$ of cold $1.8 \mathrm{~mol} / \mathrm{l}$ cushion solution (Sucrose cushion solution:sucrose cushion buffer:DTT 900:100:1) was added to the lysis solution. The mixture was transferred to a new tube preloaded with $150 \mu \mathrm{l}$ of $1.8 \mathrm{~mol} / \mathrm{l}$ sucrose cushion solution followed by centrifugation at $30,000 \mathrm{~g}$ for $45 \mathrm{~min}$. The supernatant fraction containing cytosolic components was aspirated and the nuclei were visible as a thin pellet at the bottom of the tube.

Morphometric analyses Morphometric analyses were performed using Image-Pro Plus 6.0 software (Media Cybernetics, Bethesda, MD, USA). Areas to be photographed were selected by people blind to the identity of the samples, as was scoring.

Statistical analysis Seven mice per group were studied. The measurements for each group were summarised as means $\pm \mathrm{SD}$. Image Quant 5.2 (GE Healthcare Bio-Sciences, Pittsburgh, PA, USA) was used to analyse western blots. One-way ANOVA was performed for comparisons among different groups, followed by post hoc pairwise comparisons using Tukey's test with Origin 8.6 data analysis and graphing software Lab (OriginLab, Northampton, MA, USA). In addition, we constructed a $t$ test to compare the effect of C66 on WT diabetic mice and Nrf2-null diabetic mice (Fig. 1). Differences were significant if $p<0.05$.

\section{Results}

C66 retains partial renal protection against diabetesinduced albuminuria despite deletion of the $\mathrm{Nrf} 2$ gene Diabetic WT and Nrf2-null mice had higher blood glucose levels compared with their respective controls (Fig. 1a, b). Blood glucose levels were not altered by C66 (Fig. 1a, b). Nrf2-null mice had lower systolic BP compared with WT mice (Fig. 1c), which was in line with findings reported in a previous publication [28]. Diabetes mildly increased BP (Fig. 1c) and decreased body weight (Fig. 1d) in both strains 24 weeks post diabetes. C66 had no impact on either BP or body weight (Fig. 1c, d). Diabetes increased UACR 2.57-fold in WT mice and 3.70-fold in Nrf2-null mice (Fig. 1e), suggesting the importance of NRF2 in preventing diabetic nephropathy. Protein levels of TGF- $\beta 1$ (Fig. 1f) and FN (Fig. 1g) were increased by diabetes in both strains but these elevated levels were decreased by C66. C66 decreased UACR, TGF- $\beta 1$ and FN protein by $44.72 \%, 50.80 \%$ and $48.94 \%$ in WT diabetic mice and by $21.77 \%, 21.68 \%$ and $24.83 \%$ in $N r f 2$-null diabetic mice; these effects were all significantly lower in $\mathrm{Nrf}$-null diabetic mice than in WT diabetic mice. This confirmed the requirement of NRF2 in C66 protection. On the other hand, the fact that C66 still provided protection (Fig. 1e-g) suggested the existence of an NRF2-independent protective effect of C66 against diabetic nephropathy.

C66 upregulated renal NRF2 expression via miR-200a targeting Keap1 The next study was to address whether C66 upregulates miR-200a and, if so, whether this is involved in upregulation of NRF2 function by C66. Diabetes significantly decreased renal miR-200a, while C66 increased miR200a in both control and diabetic groups (Fig. 2a). C66 decreased levels of Keap 1 mRNA (Fig. 2b) and KEAP1 protein (Fig. 2c) in the control group and also decreased diabetesinduced high levels of Keap1 mRNA and KEAP1 protein (Fig. 2b, c). Total and nuclear NRF2 protein levels were decreased in diabetic mice and increased in C66-treated control and diabetic mice (Fig. 2d, e). Immunohistochemical staining of NRF2 showed its expression to be predominantly located in the glomeruli (Fig. 2f). The C66-induced expression of nuclear NRF2 protein (Fig. 2e) was accompanied by elevated mRNA levels of Nqol (Fig. 2g) and Hol (Fig. 2h). These

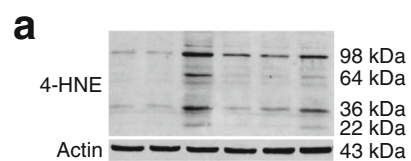

b

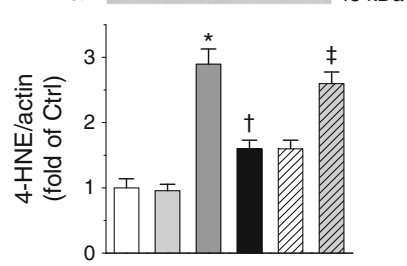

C
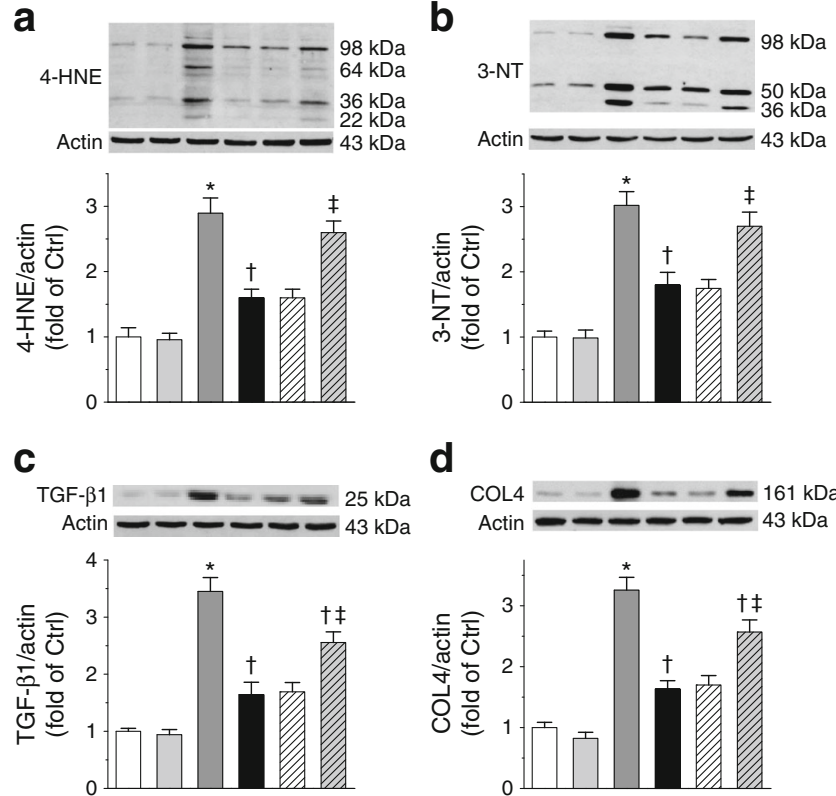

d

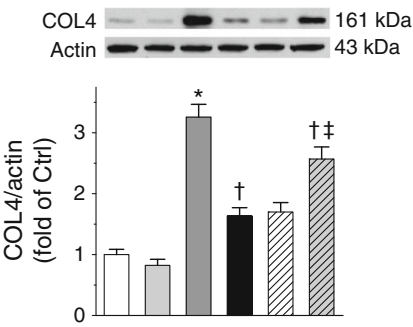

e

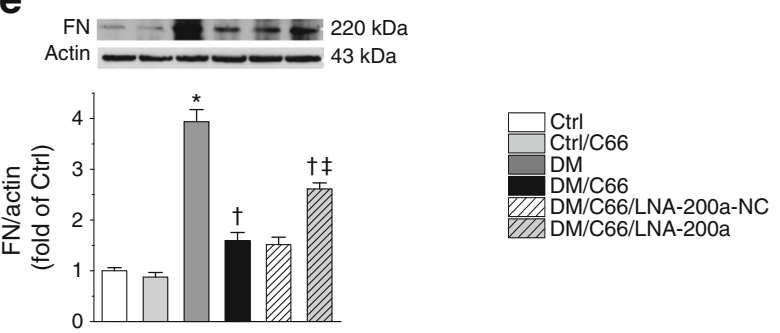

Fig. 3 LNA-200a blocks renal C66 protection against diabetic oxidative damage more effectively than $\mathrm{C} 66$ protection against diabetic renal fibrosis. Protein levels of 4-HNE (a), 3-NT (b), TGF- $\beta 1$ (c), COL4 (d) and FN (e) were evaluated by western blotting. Data were normalised by control (Ctrl) and presented as means $\pm \mathrm{SD}(n=7) .{ }^{*} p<0.05$ vs control; ${ }^{\dagger} p<0.05$ vs diabetes; ${ }^{\ddagger} p<0.05$ vs DM/C66/LNA-200a-NC 
results suggest that the induction of NRF2 expression and function by C66 may be due to stimulation of miR-200a expression. Supporting this notion, administration of LNA-200a completely prevented the actions of C66, including induction of miR-200a (Fig. 2a), suppression of diabetes induction of Keap1 mRNA and KEAP1 protein (Fig. 2b, c), prevention of diabetes reduction of total and nuclear NRF2 protein (Fig. 2d$\mathrm{f}$ ) and suppression of diabetes-induced downregulation of Nqol and Hol mRNA (Fig. 2g, h)

LNA-200a blocks renal C66 protection against diabetic oxidative damage more effectively than $\mathbf{C 6 6}$ protection against diabetic renal fibrosis, remodelling or albuminuria In the following studies, we defined which variables of diabetic nephropathy were most dependent on C66 stimulation of miR200a. As shown in Fig. 3a, b, the ability of C66 to reduce diabetes-induced high levels of 4-hydroxynonenal (4-HNE) and 3-nitrotyrosine (3-NT), indices of oxidative and nitrosative protein damage, was largely eliminated by co-administration of LNA-200a. C66 effectively reduced diabetes-increased protein levels of TGF- $\beta$, collagen 4 (COL4) and FN (Fig. 3c-e). This reduction was partially abolished by LNA-200a despite its much stronger effects on 4-HNE and 3-NT.

Significantly enlarged glomerular area (Fig. 4b), mesangial matrix expansion (Fig. 4c) and trichrome-positive area (Fig. 4d) were observed in diabetic kidney, effects that were all attenuated by $\mathrm{C} 66$ treatment. LNA-200a partially reduced the efficacy of $\mathrm{C66}$ for all three variables.

Diabetes-induced renal structural remodelling was accompanied by a slight increase in kidney size, shown by increased ratio of kidney weight to tibia length (Fig. 4e). Diabetic renal dysfunction was reflected by increased UACR at 12 and 24 weeks post diabetes and this effect was significantly reduced by C66 treatment (Fig. 4f). The protective effect of C66 on UACR was partially abolished by LNA-200a after 24 weeks of treatment (Fig. 4f).

In summary, LNA-200a completely abolished the effect of C66 on KEAP1-NRF2 signalling (Fig. 2) and oxidative damage (Fig 3a, b) but only partially abolished the protective effect of C66 against renal fibrosis (Fig. 3c-e), remodelling (Fig. 4a-d) and albuminuria (Fig. 4f). These results suggest that the C66-stimulated miR-200a-KEAP1-NRF2 pathway is
Fig. 4 C66 can still provide partial protection against diabetes-induced renal pathological changes and albuminuria despite antagonism by LNA-200a. (a) PAS and Masson's trichrome staining. Scale bar, $50 \mu \mathrm{m}$. Control, Ctrl; DM, diabetes. (b, c) Glomerular area (b) and mesangial matrix expansion (c) quantified from PAS staining. (d) Fibrosis accumulation quantified from Masson's trichrome staining. (e) Kidney weight normalised by tibia length. (f) UACR at 0,12 and 24 weeks (W). Data were normalised by $\mathrm{Ctrl}$ and presented as means $\pm \mathrm{SD}(n=7) .{ }^{*} p<0.05$ vs control; ${ }^{\dagger} p<0.05$ vs diabetes; ${ }^{\ddagger} p<0.05$ vs DM/C66/LNA-200a$\mathrm{NC}$
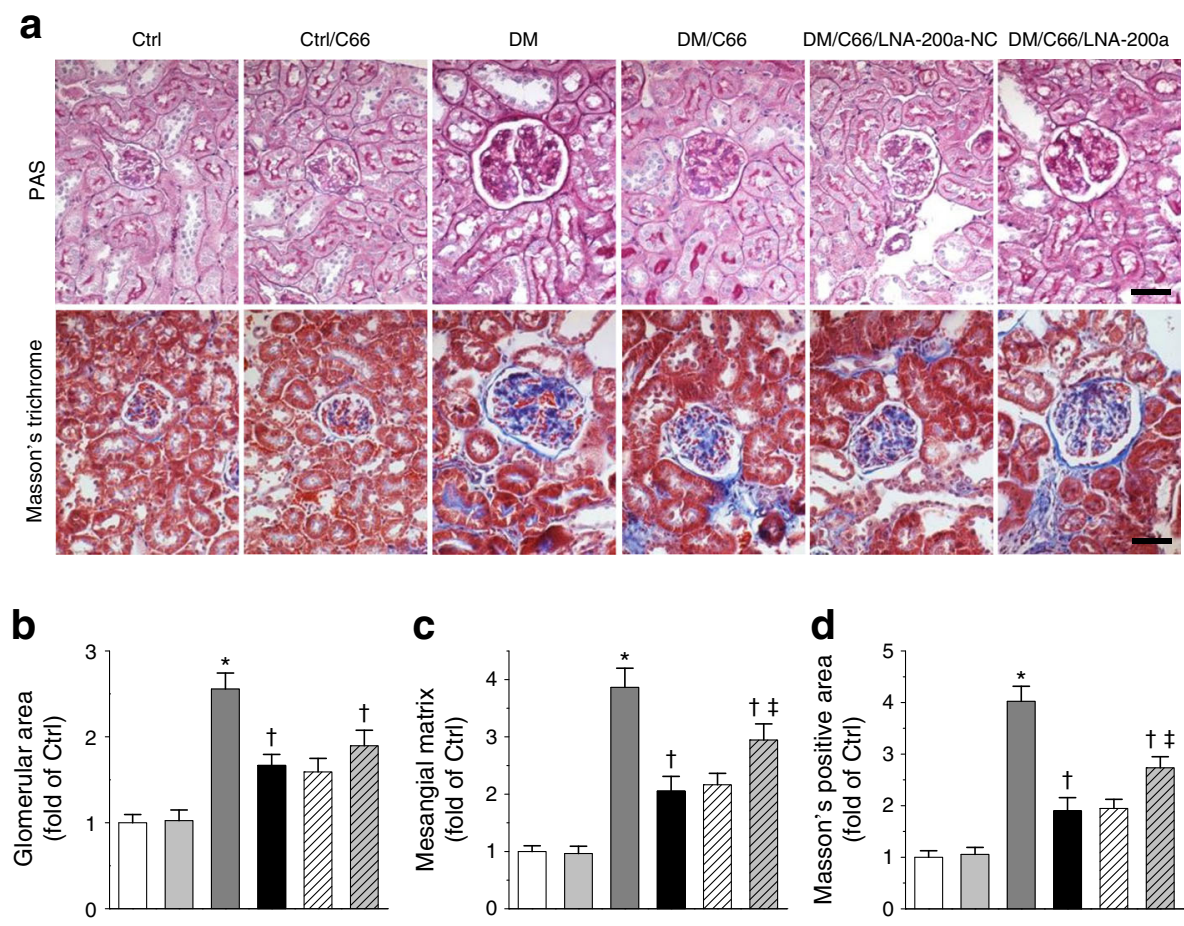

C
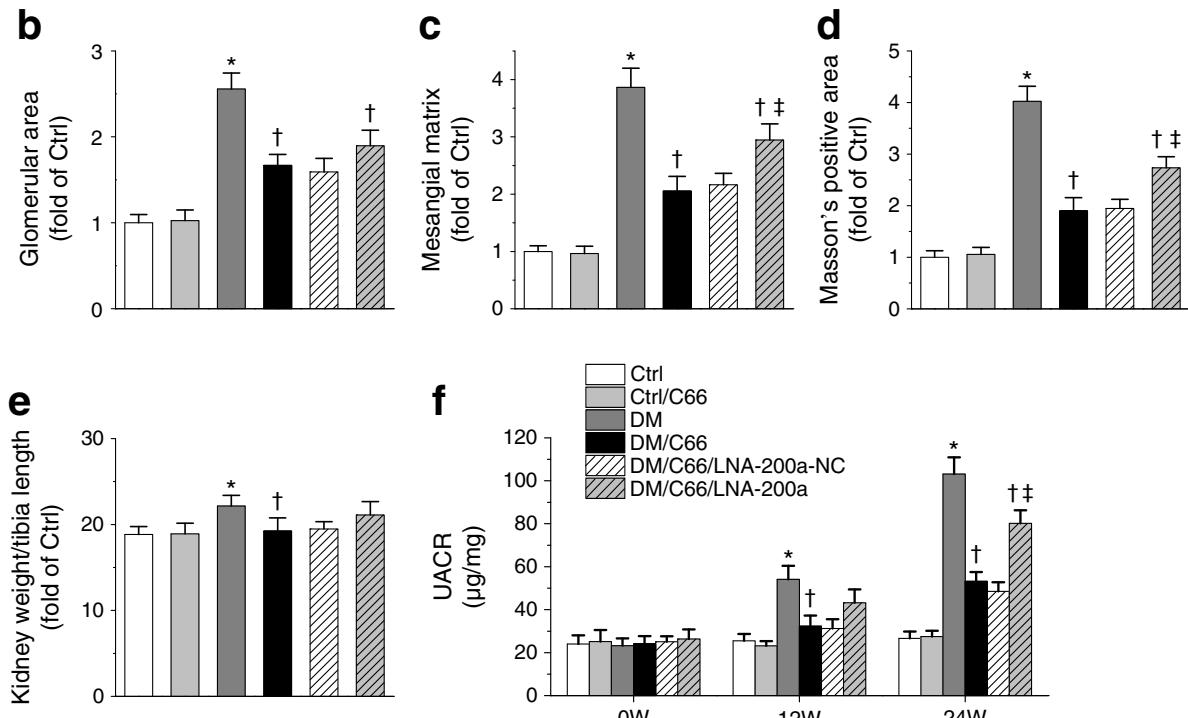
not the full explanation for $\mathrm{C} 66$ protection against diabetic nephropathy and this conclusion is consistent with the finding that $\mathrm{C} 66$ retains partial efficacy against albuminuria in diabetic Nrf2-null mice (Fig. 1e).

C66 decreased miR-21 in normal and diabetic WT mice accompanied by downregulation of p-Smad3 In diabetes, increased miR-21 downregulates its direct target, Smad7, which thereby releases Smad7 inhibition of Smad3 phosphorylation [29]. Increased phosphorylated Smad3 (p-Smad3) promotes renal fibrosis in diabetic nephropathy $[22,30]$. Since the beneficial actions of curcumin are related to downregulation of miR-21 [18, 23], we evaluated this pathway in C66 protection. As shown in Fig. 5a, b, diabetes induced pri-miR-21 and mature miR-21, and this was reversed by C66. Smad7 mRNA and Smad7 protein levels were decreased by diabetes (Fig. 5c, d) and these actions were also reversed by C66. p-Smad3 protein was markedly reduced by $\mathrm{C} 66$ under normal and diabetic conditions (Fig. 5e). These results suggest that C66 can reverse diabetes-increased $\mathrm{p}$-Smad3 levels by initially downregulating miR-21.

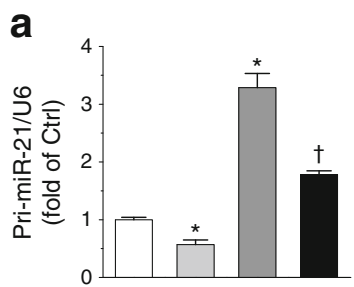

b

C

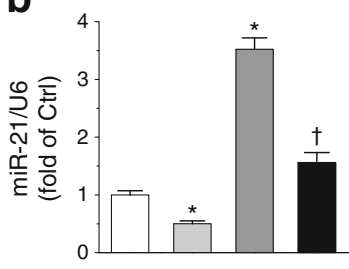

d
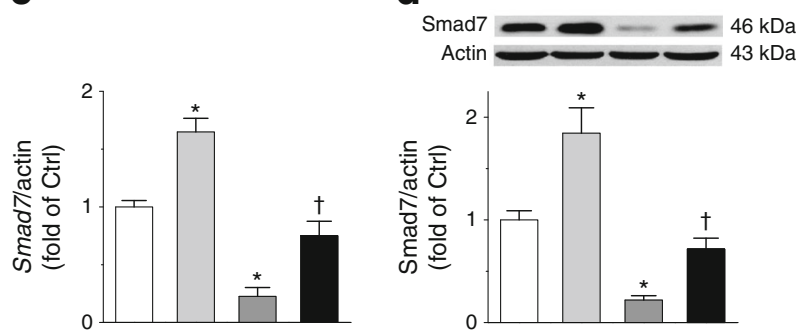

e
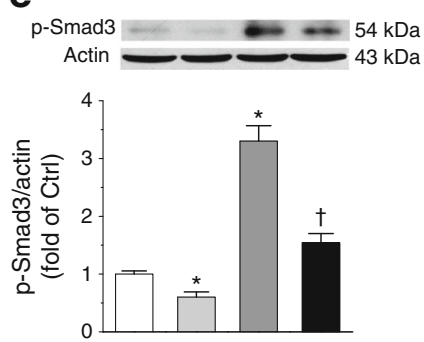

Fig. 5 C66 decreases miR-21 leading to downregulation of p-Smad3. RNA levels for pri-miR-21 (a) miR-21 (b) and $\operatorname{Smad7}$ (c) were determined by quantitative RT-PCR and protein levels of Smad7 (d) and pSmad3 (e) by western blot. Ctrl, control. White bars, Ctrl; light grey bars, Ctrl treated with C66; dark grey bars, diabetes; black bars, diabetes treated with C66. Data were normalised by Ctrl and presented as means $\pm \mathrm{SD}$ $(n=7) .{ }^{*} p<0.05$ vs control; ${ }^{\dagger} p<0.05$ vs diabetes
Both C66 and LNA-21 downregulate miR-21 in Nrf2-null diabetic mice, resulting in partial amelioration of diabetesinduced renal fibrosis To firmly establish that miR-21 inhibition is an NRF2-independent mechanism for C66 protection against diabetic nephropathy, it is necessary to test this pathway in Nrf2-null diabetic mice. To this end Nrf2-null diabetic mice were treated with C66 or LNA-21. Both C66 and LNA-21 partially, but significantly, downregulated elevated miR-21 levels in Nrf2-null diabetic mice (Fig. 6a), leading to reversal of low diabetic expression of Smad7 mRNA (Fig. 6b) and Smad7 protein (Fig. 6c). Furthermore, diabetes-increased p-Smad3 protein in Nrf2-null diabetic mice was partially reduced by both C66 and LNA-21 (Fig. 6d), along with a decrease in TGF- $\beta 1$ and FN levels (Fig. 6e, f). To further test whether c-Jun N-terminal kinase (JNK) phosphorylation is affected by C66 or LNA-21, mRNA and protein levels of $P d c d 4$, a recognised miR-21 target [31] as well as a JNK inhibitor [32], were determined. C66 and LNA-21 similarly increased the diabetes-reduced levels of $P d c d 4$ mRNA and PDCD4 protein (Fig. 6g, h), and inhibited the diabetesincreased phosphorylation of JNK (Fig. 6i).

Morphological and pathological changes were evaluated by PAS and Masson's trichrome staining (Fig. 7a). Diabetes increased glomerular area (Fig. 7b), mesangial matrix expansion (Fig. 7c) and accumulation of fibrosis (Fig. 7d) in Nrf2-null mice. Notably, these diabetes-induced changes were attenuated to a similar extent by C66 and LNA-21 (Fig 7a-d). Furthermore, UACR increased time dependently in diabetic mice and C66 and LNA-21 afforded similar, partial, attenuation of diabetes-increased UACR at 12 and 24 weeks post diabetes (Fig. 7e). These results demonstrated that in Nrf2-null diabetic mice blocking miR-21 with LNA-21 produced almost the same benefits as C66. This may indicate that all NRF2-independent benefits of $\mathrm{C} 66$ are mediated by miR-21 inhibition.

\section{Discussion}

The present study addressed whether NRF2 induction mediates, and is required for, C66 protection against diabetic nephropathy. In studies with diabetic Nrf2-null mice, we demonstrate that NRF2 plays an important role in the protection afforded by C66. By applying the miR-200a blocker, LNA-200a, we show that the miR-200a-KEAP1-NRF2 pathway enables C66 to upregulate NRF2 function. miR-21 is shown to be the basis of NRF2-independent C66 protection. In Nrf2-null mice diabetes induces miR-21 and this is suppressed by treatment with LNA21 or C66. Both C66 and LNA-21 produce similar partial renal protection in diabetic $N r f 2$-null mice. Therefore, downregulation of renal miR-21 by $\mathrm{C} 66$ in diabetic mice may be responsible for the NRF2-independent component of C66 protection against diabetic nephropathy (Fig. 8).

Oxidative stress contributes to the pathogenesis of diabetic nephropathy [33] and the KEAP1-NRF2 system even governs 
Fig. 6 Both C66 and LNA-21 downregulate miR-21 in Nrf2null mice, resulting in reduced levels of stimulators for diabetic renal fibrosis. (a, b) In Nrf2-null mice, expression levels of renal miR-21 (a) and $\operatorname{Smad} 7$ (b) RNA were evaluated by RT-PCR. (c-f) Protein levels of Smad7 (c), pSmad3 (d), TGF- $\beta 1$ (e) and FN (f) were evaluated by western blot. (g-i) Levels of $P d c d 4$ mRNA (g), PDCD4 protein (h) and JNK protein (i) were further determined. Data were normalised by $\mathrm{Ctrl}$ and presented as means $\pm \operatorname{SD}(n=7) . * p<0.05$ vs Ctrl; ${ }^{\dagger} p<0.05$ vs DM; ${ }^{\ddagger} p<0.05$ vs DM/LNA-21-NC
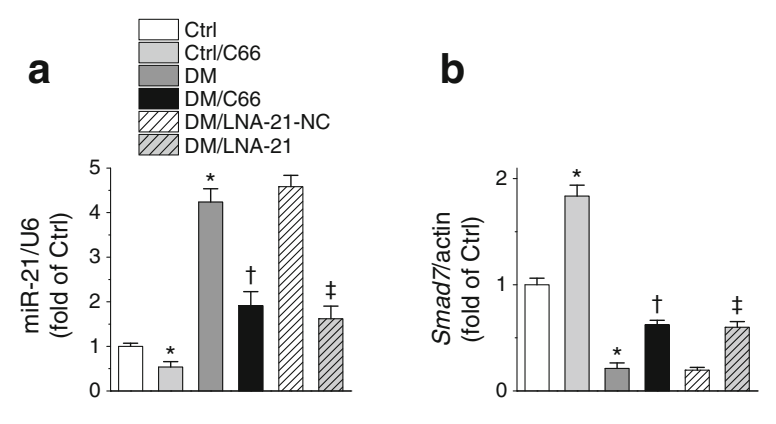

d
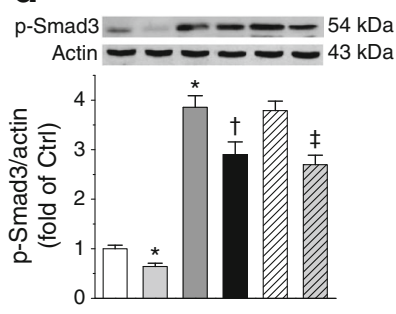

g

e
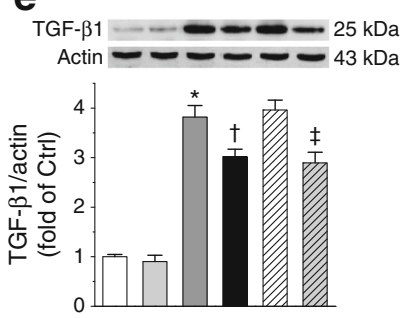

h

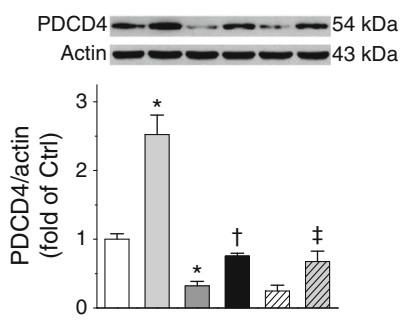

C
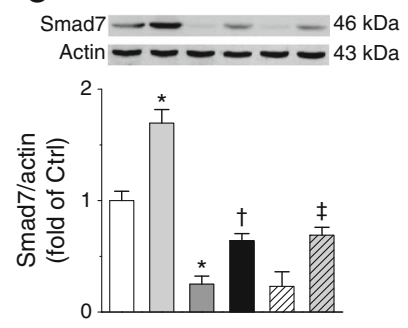

f
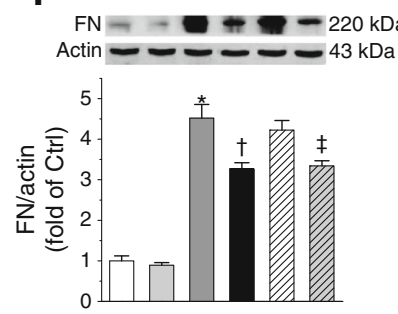

i

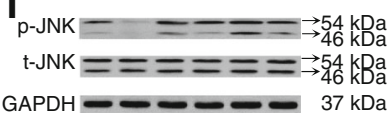

$\mathrm{GAPDH}=-\longrightarrow 37 \mathrm{kDa}$

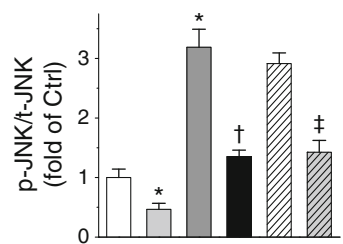

antioxidant redox signalling to prevent the initiation of diabetes mellitus $[34,35]$. We and others have demonstrated the beneficial role of NRF2 induction in the prevention of diabetic nephropathy $[2,36]$. High BP accelerates diabetic nephropathy. In the present study, the systolic BP of Nrf2-null mice was lower than that of WT mice. This result is in accordance with the finding by Erkens et al [28], which showed that elevated endothelial nitric-oxide synthase in the heart and aorta of Nrf2-null mice was the reason for the low BP. Given that high BP accelerates diabetic nephropathy whereas the Nrf2-null mice had lower BP along with severer diabetic renal injury, we may further conclude that NRF2 does play an important role in the prevention of diabetic nephropathy.

Curcumin is reported to be an activator of NRF2 [6, 37], although little is known about how this occurs. Curcumin disrupts the binding between KEAP1 and NRF2, leading to the release of NRF2 [38]. However, the fact that curcumin downregulates KEAP1 expression [8] challenges the notion that curcumin only modifies the KEAP1-NRF2 complex. Our results also demonstrate that the curcumin analogue $\mathrm{C} 66$ decreases the expression of Keapl mRNA and KEAP1 protein. Therefore, in addition to modification of the KEAP1-NRF2 complex, curcumin-decreased expression of Keapl may also account for NRF2 induction. The present study identified miR-200a as the mediator between C66 and Keap1 mRNA. miR-200a degrades Keap1 mRNA [16]. Moreover, LNA-200a exacerbates diabetic cortical and glomerular fibrogenesis and increases urinary albumin excretion [17]. Using LNA-200a, we provide the first evidence that C66 stimulates miR-200a to downregulate Keapl expression, resulting in the activation of NRF2.

C66, sulforaphane (SFN) and cinnamic aldehyde (CA) all activate NRF2. The protection from diabetic nephropathy afforded by SFN and CA is abolished in Nrf2-null diabetic mice [2]. The present study showed that $\mathrm{C} 66$ still provided significant protection in the absence of NRF2 (Fig. 1e-g). The difference between $\mathrm{C} 66$ and the other two NRF2 activators must be due to additional actions of C66. SFN and CA exclusively provide protection through NRF2 and, accordingly, no preventive effect on diabetic nephropathy is observed in Nrf2-null diabetic mice [2]. Unlike SFN and CA, we show that C66 has additional function [39] other than NRF2 upregulation [6, 37].

Curcumin is a regulator of epigenetic events, including miRNAs [40], among which miR-21 has been demonstrated to play a key role in the pathogenesis of diabetic nephropathy [20-22]. Recent findings shed light on the involvement of miR-21 as a key component of TGF- $\beta-$ Smad and PDCD4 JNK signalling. miR-21 degrades $S m a d 7$ or $P d c d 4$ mRNA to lower their protein translation [22], leading to impaired 
Fig. 7 Both C66 and LNA-21 attenuated diabetes-induced pathological changes and albuminuria in the absence of NRF2. (a-d) In Nrf2-null mice, PAS and Masson's trichrome staining (a) were performed with glomerular area (b) and mesangial matrix expansion indexes (c) quantified from PAS staining; fibrosis accumulation was reflected by Masson's-positive area (d) and was quantified from Masson's trichrome staining. Scale bar, $50 \mu \mathrm{m}$. (e) UACRs at weeks (W) 0, 12 and 24 after diabetes initiation. Data were normalised by $\mathrm{Ctrl}$ and presented as means $\pm \mathrm{SD}(n=7) . * p<0.05$ vs Ctrl; ${ }^{\dagger} p<0.05$ vs DM; ${ }^{\star} p<0.05$ vs DM/LNA-21-NC

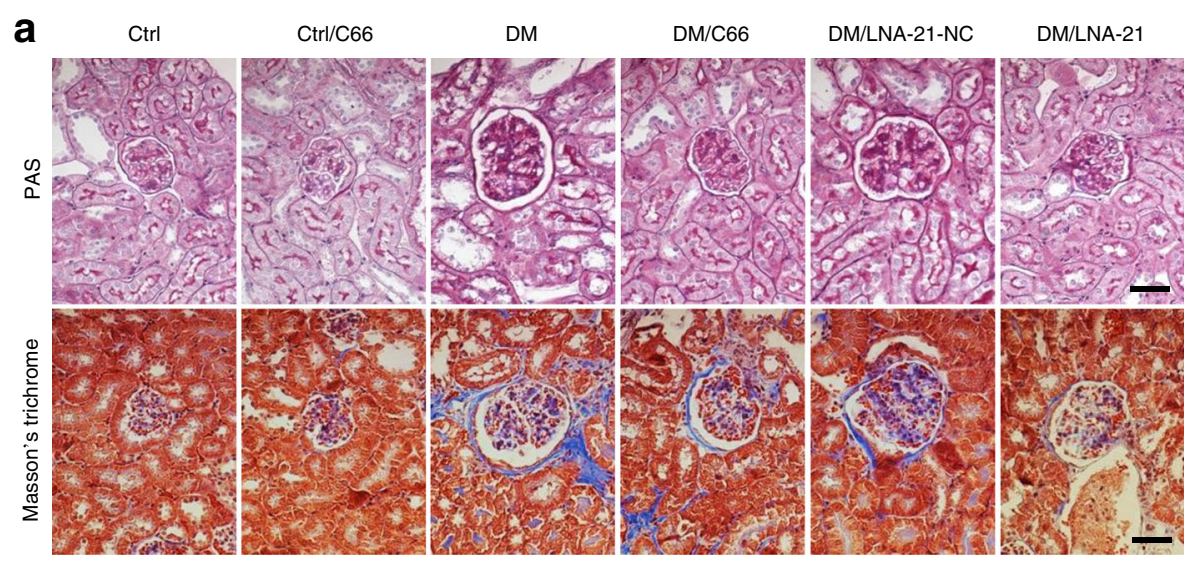

b
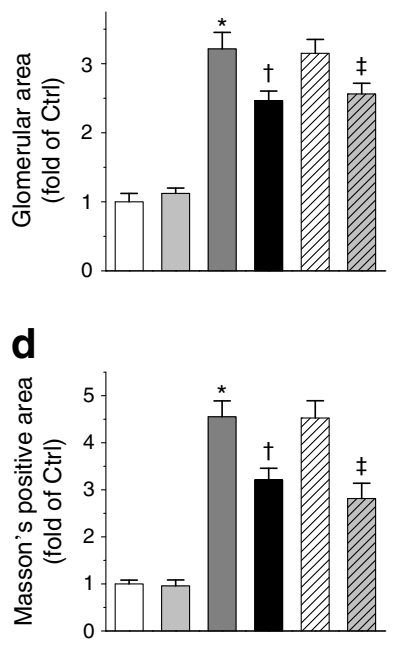

C

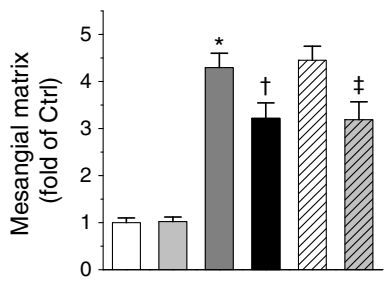

e

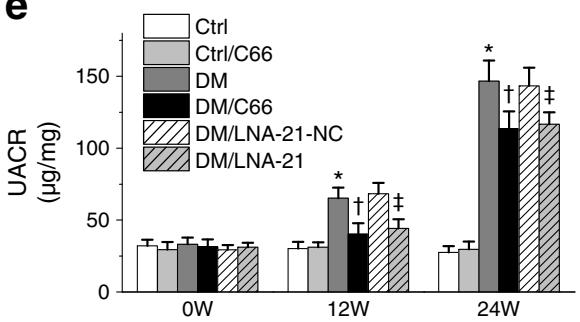

function of Smad7 or PDCD4 in the inhibition of Smad3 or JNK phosphorylation $[32,41]$. The transcription factors p-Smad3 or p-c-Jun turn on the expression of a cohort of renal fibrotic and inflammatory genes [41-45]. Therefore, inhibition of miR-21 may exert an effect different from activation of NRF2. This notion is supported by our results that knockdown of NRF2 by LNA-200a in WT mice completely abolished $\mathrm{C} 66$ protection against renal oxidative damage (Fig. 3a, b) whereas C66 still protected against renal fibrosis and kidney function (Figs 3c-e and 4d, f). Furthermore, inhibition of miR-21 by either C66 or LNA-21 negatively altered TGF- $\beta-$ Smad 3 signalling, PDCD4-JNK signalling and albuminuria even in the absence of NRF2 (Figs 6 and 7), confirming that inhibition of miR-21 offered an additional role for C66 distinct from that of inducing NRF2 function.

Inhibition of diabetic nephropathy-inducing miRNAs has attracted a great interest in recent years. miRNA inhibitors or mimics have even been studied in clinical trials [46]. The advantage of C66 compared with LNA-21 is that C66 also upregulates NRF2 in addition to knockdown of miR-21. NRF2 activation has already been applied in clinical trials.
Initial human studies of bardoxolone methyl were promising but its use was terminated in a phase III study due to heart complications [47]. However, NRF2 remains a viable drug target as demonstrated by the approval of BG-12 (dimethyl fumarate) for use in multiple sclerosis [48]. In addition to initiating treatment at an earlier stage of diabetic nephropathy and diminishing drug-drug interactions, greater attention should have been paid to the dosage of bardoxolone [49]. More recently, Tan et al showed that dh404, a derivative of bardoxolone methyl, prevented diabetic nephropathy [50], although inflammation was triggered at higher doses. These studies indicate a sweet spot for NRF2 activation [47]. Therefore the dosage of NRF2 activators should be carefully considered. The dose-effectiveness of $\mathrm{C} 66$ is very much better than native curcumin (20- to 80 -fold), and this confers a unique advantage in future clinical trials.

In summary, the present study demonstrates for the first time that C66 ameliorates diabetic nephropathy by both inhibiting miR-21 and activating NRF2 through the stimulation of miR-200a. Thus, this novel curcumin analogue with dual effects and high bioavailability urgently needs to be 


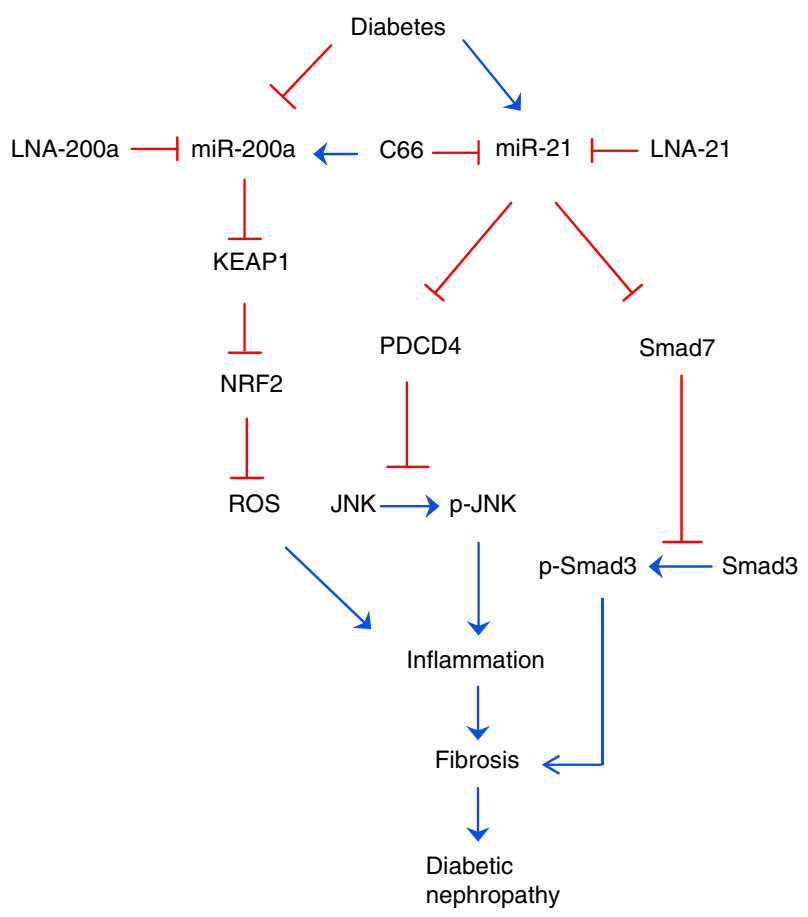

Fig. 8 Possible mechanisms for the prevention of diabetic nephropathy by C66. On one hand, C66 upregulates miR-200a to enhance NRF2 function by targeting Keap1, leading to alleviation of renal oxidative damage. On the other hand, C66 inhibits miR-21-induced activation of JNK and Smad3. These protective effects of C66 attenuate renal fibrosis and finally lead to the amelioration of diabetic nephropathy. Blue, activation; red, inhibition; ROS, reactive oxygen species

further tested in various diabetic nephropathy models and small clinical trials.

Acknowledgements Some of the data were presented as an abstract at the American Diabetes Association 75th Scientific Sessions in Boston in 2015.

Funding This work was supported in part by the National Science Foundation of China (81170669 to LM, 81200572 to GL, 2011BAI10B00 to XC), JDRF (1-INO-2014-122-A-N to YT, 1-INO2014-116-A-N to PE) and the National Institutes of Health (1R01DK 091338-01A1 to LC).

Duality of interest The authors declare that there is no duality of interest associated with this manuscript.

Contribution statement HW and LK researched the data. YT, JZ, JG, $\mathrm{PNE}$ and $\mathrm{XC}$ were involved in the experiments and acquisition of data. MK did essential statistical analysis. HW, LM, LC and GL contributed to the initial discussion and design of the project. GL provided compound C66 used in the study. LC, LM, GL, PNE and XC provided funding for this work. HW, PNE and LC wrote the manuscript. LK, YT, PNE, JZ, JG, GL, MK, XC, LM and LC reviewed and revised the manuscript. YT, PNE and $\mathrm{XC}$ were involved in discussion of the manuscript. All the authors approve the version to be published. LC and LM are guarantors of this work and, as such, had full access to all the data in the study and take responsibility for the integrity of the data and the accuracy of the data analysis.

\section{References}

1. Dronavalli S, Duka I, Bakris GL (2008) The pathogenesis of diabetic nephropathy. Nat Clin Pract Endocrinol Metab 4:444-452

2. Zheng H, Whitman SA, Wu W et al (2011) Therapeutic potential of Nrf2 activators in streptozotocin-induced diabetic nephropathy. Diabetes 60:3055-3066

3. Miyata T, Suzuki N, van Ypersele de Strihou C (2013) Diabetic nephropathy: are there new and potentially promising therapies targeting oxygen biology? Kidney Int 84:693-702

4. Jiang T, Huang Z, Lin Y, Zhang Z, Fang D, Zhang DD (2010) The protective role of Nrf2 in streptozotocin-induced diabetic nephropathy. Diabetes 59:850-860

5. Nguyen T, Sherratt PJ, Pickett CB (2003) Regulatory mechanisms controlling gene expression mediated by the antioxidant response element. Annu Rev Pharmacol Toxicol 43:233-260

6. Kang ES, Woo IS, Kim HJ et al (2007) Up-regulation of aldose reductase expression mediated by phosphatidylinositol 3-kinase/ Akt and Nrf2 is involved in the protective effect of curcumin against oxidative damage. Free Radic Biol Med 43:535-545

7. Huang J, Huang K, Lan T et al (2013) Curcumin ameliorates diabetic nephropathy by inhibiting the activation of the SphK1-S1P signaling pathway. Mol Cell Endocrinol 365:231-240

8. Soetikno V, Sari FR, Lakshmanan AP et al (2013) Curcumin alleviates oxidative stress, inflammation, and renal fibrosis in remnant kidney through the Nrf2-keap1 pathway. Mol Nutr Food Res 57: 1649-1659

9. Li D, Lu Z, Jia J, Zheng Z, Lin S (2013) Curcumin ameliorates Podocytic adhesive capacity damage under mechanical stress by inhibiting miR-124 expression. Kidney Blood Press Res 38:61-71

10. Soetikno V, Watanabe K, Sari FR et al (2011) Curcumin attenuates diabetic nephropathy by inhibiting PKC- $\alpha$ and PKC- $\beta_{1}$ activity in streptozotocin-induced type I diabetic rats. Mol Nutr Food Res 55: $1655-1665$

11. Tikoo K, Meena RL, Kabra DG, Gaikwad AB (2008) Change in post-translational modifications of histone $\mathrm{H} 3$, heat-shock protein27 and MAP kinase p38 expression by curcumin in streptozotocininduced type I diabetic nephropathy. Br J Pharmacol 153:12251231

12. Pan Y, Wang Y, Zhao Y et al (2014) Inhibition of JNK phosphorylation by a novel curcumin analog prevents high glucose-induced inflammation and apoptosis in cardiomyocytes and the development of diabetic cardiomyopathy. Diabetes 63:3497-3511

13. Pan Y, Zhang X, Wang Y et al (2013) Targeting JNK by a new curcumin analog to inhibit NF-kB-mediated expression of cell adhesion molecules attenuates renal macrophage infiltration and injury in diabetic mice. PLoS One 8:e79084

14. Liu Y, Wang Y, Miao X et al (2014) Inhibition of JNK by compound C66 prevents pathological changes of the aorta in STZinduced diabetes. J Cell Mol Med 18:1203-1212

15. Bhatt K, Mi QS, Dong Z (2011) microRNAs in kidneys: biogenesis, regulation, and pathophysiological roles. Am J Physiol Renal Physiol 300:F602-F610

16. Eades G, Yang M, Yao Y, Zhang Y, Zhou Q (2011) miR-200a regulates Nrf2 activation by targeting Keap1 mRNA in breast cancer cells. J Biol Chem 286:40725-40733

17. Wei J, Zhang Y, Luo Y et al (2013) Aldose reductase regulates miR$200 a-3 p / 141-3 p$ to coordinate Keap1-Nrf2, Tgf $\beta 1 / 2$, and Zeb1/2 signaling in renal mesangial cells and the renal cortex of diabetic mice. Free Radic Biol Med 67C:91-102

18. Ali S, Ahmad A, Banerjee S et al (2010) Gemcitabine sensitivity can be induced in pancreatic cancer cells through modulation of miR-200 and miR-21 expression by curcumin or its analogue CDF. Cancer Res 70:3606-3617 
19. Soubani O, Ali AS, Logna F, Ali S, Philip PA, Sarkar FH (2012) Re-expression of miR-200 by novel approaches regulates the expression of PTEN and MT1-MMP in pancreatic cancer. Carcinogenesis 33:1563-1571

20. Dey N, Das F, Mariappan MM et al (2011) MicroRNA-21 orchestrates high glucose-induced signals to TOR complex 1, resulting in renal cell pathology in diabetes. J Biol Chem 286:25586-25603

21. Wang JY, Gao YB, Zhang N et al (2014) miR-21 overexpression enhances TGF- $\beta 1$-induced epithelial-to-mesenchymal transition by target smad7 and aggravates renal damage in diabetic nephropathy. Mol Cell Endocrinol 392:163-172

22. Zhong X, Chung AC, Chen HY et al (2013) miR-21 is a key therapeutic target for renal injury in a mouse model of type 2 diabetes. Diabetologia 56:663-674

23. Mudduluru G, George-William JN, Muppala S et al (2011) Curcumin regulates miR-21 expression and inhibits invasion and metastasis in colorectal cancer. Biosci Rep 31:185-197

24. Zhang Z, Chen J, Zhou S et al (2015) Magnolia bioactive constituent 4-O-methylhonokiol prevents the impairment of cardiac insulin signaling and the cardiac pathogenesis in high-fat diet-induced obese mice. Int J Biol Sci 11:879-891

25. Cai L, Wang J, Li Y et al (2005) Inhibition of superoxide generation and associated nitrosative damage is involved in metallothionein prevention of diabetic cardiomyopathy. Diabetes 54:1829-1837

26. Wang Y, Feng W, Xue W et al (2009) Inactivation of GSK-3 $\beta$ by metallothionein prevents diabetes-related changes in cardiac energy metabolism, inflammation, nitrosative damage, and remodeling. Diabetes 58:1391-1402

27. Wu H, Zhou S, Kong L et al (2014) Metallothionein deletion exacerbates intermittent hypoxia-induced renal injury in mice. Toxicol Lett 232:340-348

28. Erkens R, Kramer CM, Luckstadt W et al (2015) Left ventricular diastolic dysfunction in Nrf2 knock out mice is associated with cardiac hypertrophy, decreased expression of SERCA2a, and preserved endothelial function. Free Radic Biol Med 89:906-917

29. Nakao A, Okumura K, Ogawa H (2002) Smad7: a new key player in TGF- $\beta$-associated disease. Trends Mol Med 8:361-363

30. Wang JY, Gao YB, Zhang N et al (2014) Tongxinluo ameliorates renal structure and function by regulating miR-21-induced epithelial-to-mesenchymal transition in diabetic nephropathy. Am J Physiol Renal Physiol 306:F486-F495

31. Frankel LB, Christoffersen NR, Jacobsen A, Lindow M, Krogh A, Lund AH (2008) Programmed cell death 4 (PDCD4) is an important functional target of the microRNA miR-21 in breast cancer cells. J Biol Chem 283:1026-1033

32. Bitomsky N, Bohm M, Klempnauer KH (2004) Transformation suppressor protein Pdcd4 interferes with JNK-mediated phosphorylation of c-Jun and recruitment of the coactivator p300 by c-Jun. Oncogene 23:7484-7493

33. Keshari KR, Wilson DM, Sai V et al (2014) Non-invasive in vivo imaging of diabetes-induced renal oxidative stress and response to therapy using hyperpolarized $13 \mathrm{C}$ dehydroascorbate magnetic resonance. Diabetes 64:344-352
34. Uruno A, Furusawa Y, Yagishita Y et al (2013) The Keap1-Nrf2 system prevents onset of diabetes mellitus. Mol Cell Biol 33:29963010

35. Yagishita Y, Fukutomi T, Sugawara A et al (2014) Nrf2 protects pancreatic $\beta$-cells from oxidative and nitrosative stress in diabetic model mice. Diabetes 63:605-618

36. Li B, Cui W, Tan Y et al (2014) Zinc is essential for the transcription function of $\mathrm{Nrf} 2$ in human renal tubule cells in vitro and mouse kidney in vivo under the diabetic condition. J Cell Mol Med 18: 895-906

37. Balogun E, Hoque M, Gong P et al (2003) Curcumin activates the haem oxygenase- 1 gene via regulation of $\mathrm{Nrf} 2$ and the antioxidantresponsive element. Biochem J 371:887-895

38. Esatbeyoglu T, Huebbe P, Ernst IM, Chin D, Wagner AE, Rimbach G (2012) Curcumin-from molecule to biological function. Angew Chem Int Ed Engl 51:5308-5332

39. Soetikno V, Suzuki K, Veeraveedu PT et al (2013) Molecular understanding of curcumin in diabetic nephropathy. Drug Discov Today 18:756-763

40. Teiten MH, Dicato M, Diederich M (2013) Curcumin as a regulator of epigenetic events. Mol Nutr Food Res 57:1619-1629

41. Chen HY, Huang XR, Wang W et al (2011) The protective role of Smad7 in diabetic kidney disease: mechanism and therapeutic potential. Diabetes 60:590-601

42. Chung AC, Zhang H, Kong YZ et al (2010) Advanced glycation end-products induce tubular CTGF via TGF- $\beta$-independent Smad3 signaling. J Am Soc Nephrol 21:249-260

43. Ai J, Nie J, He J et al (2014) GQ5 hinders renal fibrosis in obstructive nephropathy by selectively inhibiting TGF- $\beta$-induced Smad 3 phosphorylation. J Am Soc Nephrol 26:1827-1838

44. Ishida T, Haneda M, Maeda S, Koya D, Kikkawa R (1999) Stretchinduced overproduction of fibronectin in mesangial cells is mediated by the activation of mitogen-activated protein kinase. Diabetes 48:595-602

45. Bennett BL, Sasaki DT, Murray BW et al (2001) SP600125, an anthrapyrazolone inhibitor of Jun N-terminal kinase. Proc Natl Acad Sci U S A 98:13681-13686

46. Wahid F, Shehzad A, Khan T, Kim YY (2010) MicroRNAs: synthesis, mechanism, function, and recent clinical trials. Biochim Biophys Acta 1803:1231-1243

47. Hall ET, Bhalla V (2014) Is there a sweet spot for Nrf2 activation in the treatment of diabetic kidney disease? Diabetes 63:2904-2905

48. Gold R, Kappos L, Arnold DL et al (2012) Placebo-controlled phase 3 study of oral BG-12 for relapsing multiple sclerosis. N Engl J Med 367:1098-1107

49. Ellison DH (2014) Bardoxolone methyl in type 2 diabetes and advanced chronic kidney disease. N Engl J Med 370:1768

50. Tan SM, Sharma A, Stefanovic N et al (2014) Derivative of bardoxolone methyl, dh404, in an inverse dose-dependent manner lessens diabetes-associated atherosclerosis and improves diabetic kidney disease. Diabetes 63:3091-3103 